\title{
The Use of HbA1c, Glycated Albumin and Continuous Glucose Monitoring to Assess Glucose Control in the Chronic Kidney Disease Population Including Dialysis
}

\author{
Tobias Bomholt $^{\mathrm{a}}$ Therese Adrian ${ }^{\mathrm{a}}$ Kirsten Nørgaard ${ }^{\mathrm{b}}$ Ajenthen G. Ranjan ${ }^{\mathrm{b}, \mathrm{c}}$ \\ Thomas Almdal $^{d}$ Anders Larsson $^{\mathrm{e}}$ Mette Vadstrup ${ }^{\mathrm{a}}$ Marianne Rix ${ }^{\mathrm{a}}$ \\ Bo Feldt-Rasmussen ${ }^{a}$ f Mads Hornum ${ }^{a, f}$ \\ aDepartment of Nephrology, Rigshospitalet, University of Copenhagen, Copenhagen, Denmark; bSteno Diabetes \\ Center Copenhagen, University of Copenhagen, Copenhagen, Denmark; 'Danish Diabetes Academy, Odense, \\ Denmark; ${ }^{d}$ Department of Endocrinology, Rigshospitalet, University of Copenhagen, Copenhagen, Denmark; \\ eDepartment of Medical Sciences, Clinical Chemistry, Uppsala University, Uppsala, Sweden; fDepartment of Clinical \\ Medicine, Faculty of Health and Medical Sciences, University of Copenhagen, Copenhagen, Denmark
}

\section{Keywords}

Diabetes - Glycated albumin - Continuous glucose monitoring $\cdot$ Chronic kidney disease $\cdot$ Dialysis

\begin{abstract}
Background: Glycated haemoglobin $\mathrm{A}_{1 \mathrm{c}}\left(\mathrm{HbA}_{1 \mathrm{c}}\right)$ has limitations as a glycemic marker for patients with diabetes and CKD and for those receiving dialysis. Glycated albumin is an alternative glycemic marker, and some studies have found that glycated albumin more accurately reflects glycemic control than $\mathrm{HbA}_{1 c}$ in these groups. However, several factors are known to influence the value of glycated albumin including proteinuria. Continuous glucose monitoring (CGM) is another alternative to $\mathrm{HbA}_{1 \mathrm{c}}$. CGM allows one to assess mean glucose, glucose variability, and the time spent in hypo-, normo-, and hyperglycemia. Currently, several different CGM models are approved for use in patients receiving dialysis; CKD (not on dialysis) is not a contraindication in any of these models. Some devices are for blind recording, while others provide real-time data to patients. Small studies suggest that
\end{abstract}

karger@karger.com

(c) 2020 S. Karger AG, Basel

www.karger.com/nef

Karger'
CGM could improve glycemic control in hemodialysis patients, but this has not been studied for individual CKD stages. Summary: Glycated albumin and CGM avoid the pitfalls of $\mathrm{HbA}_{1 \mathrm{c}}$ in $\mathrm{CKD}$ and dialysis populations. However, the value of glycated albumin may be affected by several factors. CGM provides a precise estimation of the mean glucose. Here, we discuss the strengths and limitations for using $\mathrm{HbA1c}$, glycated albumin, or CGM in CKD and dialysis population. Key Messages: Glycated albumin is an alternative glycemic marker but is affected by proteinuria. CGM provides a precise estimation of mean glucose and glucose variability. It remains unclear if CGM improves glycemic control in the CKD and dialysis populations.

(c) 2020 S. Karger AG, Basel

\section{Introduction}

Glycated haemoglobin $\mathrm{A} 1 \mathrm{c}\left(\mathrm{HbA}_{1 \mathrm{c}}\right)$ and self-monitoring of blood glucose (SMBG) are cornerstones of glycemic monitoring in patients with diabetes. $\mathrm{HbA}_{1 \mathrm{c}}$ is used 
as a retrospective measurement of mean blood glucose over the previous 9-12 weeks [1]. $\mathrm{HbA}_{1 \mathrm{c}}$ is a product of the covalent and irreversible binding of plasma glucose molecules to hemoglobin. The normal range of $\mathrm{HbA}_{1 \mathrm{c}}$ and its correlation with the estimated mean blood glucose were determined from studies on patients without severe $\mathrm{CKD}$ [1]. $\mathrm{HbA}_{1 \mathrm{c}}$ values have been assessed in hemodialysis patients using glycated albumin and continuous glucose monitoring (CGM) as a reference, and these studies indicate that $\mathrm{HbA}_{1 c}$ significantly underestimates mean blood glucose in these patients $[2,3]$.

The interpretation of $\mathrm{HbA}_{1 \mathrm{c}}$ in relation to glycemic control is based on the assumption that the erythrocyte lifespan is the same for all patients. This means that the time available for glycation of hemoglobin is identical. Several studies have found a reduced erythrocyte lifespan in patients on hemodialysis and proposed this as a key mechanism for lowering $\mathrm{HbA}_{1 \mathrm{c}}$ because the glycation of hemoglobin is a continuous process throughout the erythrocyte lifespan [4]. In addition, erythropoiesis-stimulating agents used to treat nephrogenic anemia will increase the erythropoiesis and thereby the proportion of young non-glycated erythrocytes. This is thought to lower the $\mathrm{HbA}_{1 \mathrm{c}}$ value. Only a few small studies have validated $\mathrm{HbA}_{1 \mathrm{c}}$ in patients with diabetes receiving peritoneal dialysis. This makes it difficult to assess the validity of $\mathrm{HbA}_{1 \mathrm{c}}$ in this population [5].

The latest guidelines from Kidney Disease Improving Global Outcomes emphasize the uncertainty of $\mathrm{HbA}_{1 \mathrm{c}}$, and the use of CGM is suggested as an alternative [6]. However, experience with CGM in patients with CKD and on dialysis is limited in both research and clinical settings. In this mini-review, we discuss the use of glycated albumin as glycemic marker in the CKD- and dialysis population. In addition, we review current guidelines on CGM and discuss strengths and limitations for using CGM.

\section{Glycated Albumin}

Plasma albumin constitutes $60 \%$ of plasma proteins and plays a key role in the maintenance of the colloid osmotic pressure in plasma. It also serves as a binding site and carrier for endogenous and exogenous components including many metals, hormones, and pharmaceuticals. Glycated albumin is formed when blood glucose is covalently bound to a free amino group on albumin. The degree of glycated albumin corresponds to the mean blood glucose for the previous 3 weeks reflecting albumin's half-

CGM and Glycated Albumin in CKD

Including Dialysis life of approximately 3 weeks [7]. Glycated albumin is an intermediate marker of glycemic control and is more sensitive to changes in mean blood glucose versus $\mathrm{HbA}_{1 c}$ which can be an advantage in terms of assessing the response to treatment changes.

The different hematological factors that affect $\mathrm{HbA}_{1 \mathrm{c}}$ do not affect glycated albumin, and studies with patients on dialysis suggest that glycated albumin is more accurate than $\mathrm{HbA}_{1 \mathrm{c}}$ in the assessment of glycemic status [8]. Like $\mathrm{HbA}_{1 \mathrm{c}}$, the concentration of blood glucose and the reaction time between albumin and blood glucose are determining factors for the degree to which albumin is glycated. Thus, factors that affect the half-life of albumin will affect the level of glycated albumin and thus the correlation to mean blood glucose during the previous 3 weeks.

An increased half-life of albumin is found in patients with cirrhosis resulting in elevated levels of glycated albumin [9]. A reduced albumin half-life has been seen in patients with severe proteinuria, where nephrogenic loss of albumin induces a compensatory increase in hepatic albumin synthesis that increases the turnover for albumin and lowers the value of glycated albumin [10]. Proteinuria is common in subjects with diabetes and late stages of CKD; thus, this error is of concern. In addition, patients receiving peritoneal dialysis can potentially lose a substantial amount of albumin from the peritoneum, and this albumin loss can also affect the degree of albumin glycation.

Glycated albumin can be measured by ion exchange chromatography, high-performance liquid chromatography, and enzymatic assays. Glycated albumin is reported as the percentage of albumin that is bound to glucose. However, there is no international consensus on the reference interval for normal values of glycated albumin nor a standardized method similar to the approached used for $\mathrm{HbA}_{1 c}$, where high-performance liquid chromatography is the accepted method [11]. Currently, the reference intervals differ depending on the measurement method [7]. Although enzymatic assays of glycated albumin have been extensively studied, there is no universally accepted reference interval for this method.

In one study examining glycated albumin in individuals with diabetes (1,559 subjects not on dialysis), a glycated albumin value of $18 \%$ was the diagnostic cutoff value for diabetes when compared with $\mathrm{HbA}_{1 \mathrm{c}}[12]$. However, another study found a cutoff value of $14.3 \%$ [13]. The lack of an internationally accepted reference interval and cutoff value for diabetes makes it difficult to implement glycated albumin clinically and in research. In addition, studies have not sufficiently examined glycated al- 
Table 1. Key features of CGM models [19-25]

\begin{tabular}{|c|c|c|c|c|c|c|c|}
\hline & $\begin{array}{l}\text { Abbott FreeStyle } \\
\text { Libre [19] }\end{array}$ & $\begin{array}{l}\text { Abbott FreeStyle } \\
\text { Libre Pro }\end{array}$ & $\begin{array}{l}\text { Dexcom } \\
\text { G6 [20] }\end{array}$ & $\begin{array}{l}\text { Medtronic } \\
\text { Ipro2 [21] }\end{array}$ & $\begin{array}{l}\text { Medtronic Envision } \\
\text { Pro }[22,23]\end{array}$ & $\begin{array}{l}\text { Medtronic Guardian } \\
\text { Connect [24] }\end{array}$ & $\begin{array}{l}\text { Eversense } \\
\text { XL [25] }\end{array}$ \\
\hline Approved for CKD (not on dialysis) & Yes & Yes & Yes & Yes & Yes & Yes & Yes \\
\hline Approved for peritoneal dialysis & No & No & No & Yes & Yes & Yes & Yes \\
\hline Open CGM & Yes & No & Yes & No & No & Yes & Yes \\
\hline Blinded CGM & No & Yes & Yes* & Yes & Yes & No & No \\
\hline Sensor interval, $\mathrm{mmol} / \mathrm{L}$ & $2.2-27.8$ & $2.2-27.8$ & $2.2-22.2$ & $2.2-22.2$ & $2.2-22.2$ & $2.2-22.2$ & $2.2-22.2$ \\
\hline Accuracy (MARD), \% & 9.4 & 12.3 & 9.0 & 11.0 & 11.0 & 8.7 & 11.6 \\
\hline
\end{tabular}

CGM, continuous glucose monitoring; MARD, mean absolute relative difference. ${ }^{*}$ Can be adjusted for both open and blinded use.

bumin prospectively and whether it can be used to improve glycemic control or reduce microvascular complications as an independent glycemic marker.

\section{CGM: Available Models and Interpretation}

A CGM device uses an intradermal sensor to measure interstitial glucose levels. The result is an indirect measurement of blood glucose because the interstitial glucose and blood glucose equilibrate within minutes. The sensor measures glucose enzymatically (glucose oxidase) except for the Eversense XL CGM that uses fluorescence.

CGM models can be divided into blinded and open CGM models (Table 1). The blinded models are usually read by the clinician and are suitable for research or status assessment because the results do not affect the patient's behavior in relation to diet or anti-diabetic treatment. The open CGM models allow the user to read the glucose values continuously. The open CGM models can be further divided into real-time CGM and intermittently viewed CGM (Table 1). Real-time CGM automatically transmits interstitial glucose values to the user enabling constant glucose monitoring. The CGM model FreeStyle Libre is an intermittently viewed CGM and shows both the current glucose value and the 8-hour retrospective glucose values when the sensor is swiped by a receiver unit.

Currently, 4 CGM models are allowed for use in hemodialysis and peritoneal dialysis patients (Table 1). These are the Eversense XL, Ipro2, Guardian Connect, and the Envision Pro shown in Figure 1. For patients with $\mathrm{CKD}$ (not on dialysis), no contraindications are provided by the manufacturer for the use of CGM.

\section{Interpretation of CGM}

The latest international guidelines on CGM for analysis and treatment goals were published in 2019 [14]. A CGM period of at least 14 days is recommended with active measurements for $70 \%$ of the time for precise calculation of the mean sensor glucose. The glucose management indicator can be calculated from the mean sensor for an estimated HbA1c [14]. CGM analysis also allows for the assessment of glucose variability, which describes the fluctuations in interstitial glucose values and is measured by the coefficient of variation $(\% \mathrm{CV})$ with a value $\geq 36 \%$ indicating an increased risk of hypoglycemia [14].

Another key metric is time-in-range, which is the percentage of time spent in an interstitial glucose range between 3.9 and $10.0 \mathrm{mmol} / \mathrm{L}$. The time-above-range and time-below-range are the percentages of time spent above and below this range, respectively. Patients with type 1 and type 2 diabetes with CKD should maintain the following: time-in-range $>50 \%$, time-below-range $<1 \%$, time-above-range $<50 \%$ (glucose range $>10.0 \mathrm{mmol} / \mathrm{L}$ ), and $<10 \%$ of time in glucose range $>13.9 \mathrm{mmol} / \mathrm{L}$. However, these recommendations do not specifically include patients on dialysis [14].

When assessing CGM data, it is important to recognize that the accuracy varies between each CGM model. The most commonly used metric for assessment of CGM accuracy is the mean absolute relative difference (MARD) (Table 1). A low MARD value indicates high CGM accuracy and vice versa. However, MARD varies across glucose ranges with high MARD values in hypoglycemia and low MARD values in hyperglycemia. Thus, MARD is often reported both as a total value and a stratified value across predefined glucose ranges. 


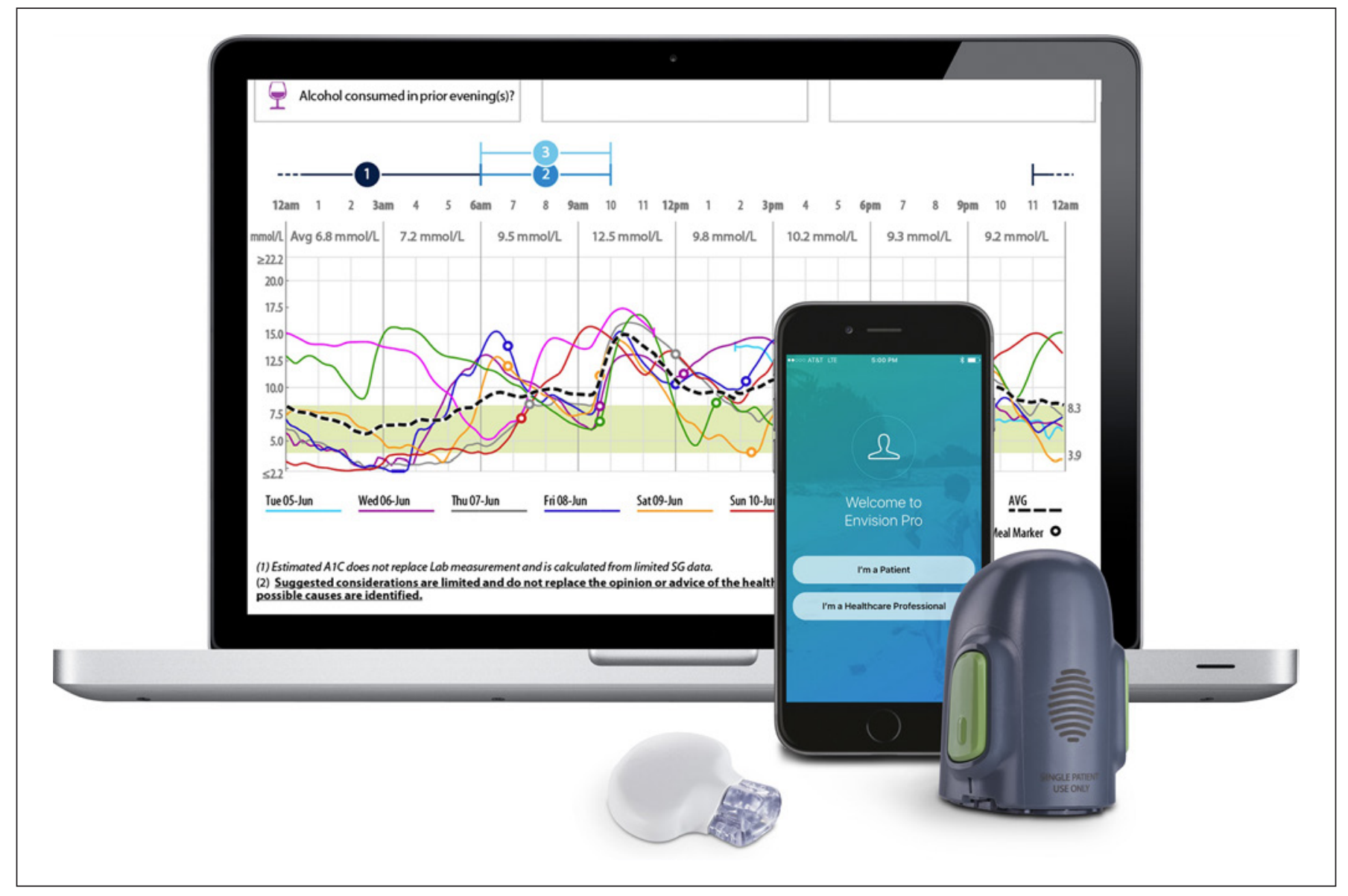

Fig. 1. The Envision Pro sensor is a blinded CGM model with a sensor applied to the skin using the serter. A recorder unit is connected to the sensor and can transmit glucose measurements to the receiver unit (e.g., a smart phone). Data report from the Envision
Pro provides information on mean interstitial glucose values, which are reported for each day and for the entire period of monitoring. In addition, metrics for glycemic variability and time-inranges are reported. CGM, continuous glucose monitoring.

\section{Impact of CGM on Glycemic Control on the CKD and Dialysis Populations}

The clinical use of CGM in patients receiving hemodialysis or peritoneal dialysis has not been well studied, and it is unclear whether the use of CGM can improve glycemic control or reduce the risk of hypoglycemia in this population. Two studies have evaluated the effect of CGM on glycemic control in the hemodialysis population where a clinician used CGM readings to adjust the anti-diabetic treatment. Képénékian et al. [15] reported that participants wore a real-time CGM device for a total of $54 \mathrm{~h}$ at baseline $(n=$ 28) with a follow-up period of 3 months. Insulin therapy was adjusted according to the CGM values. At follow-up, $\mathrm{HbA}_{\mathrm{lc}}$ was reduced from $65 \pm 11 \mathrm{mmol} / \mathrm{mol}$ to $60 \pm 11$ $\mathrm{mmol} / \mathrm{mol}$ without an increase in hypoglycemia [15].

CGM and Glycated Albumin in CKD Including Dialysis
Joubert et al. [16] studied participants with type 2 diabetes undergoing hemodialysis $(n=15)$ using SMBG for 6 weeks with measurements 3 times daily during a 6 -week period followed by another 6-week period with intermittent CGM measurements performed for 5 days every 2 weeks. SMBG and CGM values were used to adjust the treatment. They found that glycemic monitoring by SMBG did not improve glycemic control assessed by $\mathrm{HbA}_{1 \mathrm{c}}$ in comparison with CGM monitoring. This was associated with a more frequent adjustment of the antidiabetic treatment and a reduction of $\mathrm{HbA}_{1 \mathrm{c}}$ from $51 \pm 11$ $\mathrm{mmol} / \mathrm{mol}$ to $47 \pm 11 \mathrm{mmol} / \mathrm{mol}$.

Implementing daily use of an open CGM model is more challenging for the most debilitated patients on dialysis because these models require independent handling of the receiver unit, application of a new sensor, and 
in some models daily SMBG for calibration. Alternatively, the blinded type of CGM may be used periodically under guidance from dialysis staff. For example, this could be part of the routine status assessment performed every 6-12 months for patients undergoing hemodialysis.

There are no studies evaluating the clinical effect of CGM on the individual stages of CKD. In the early stages of CKD (CKD stages 1-3), the limited effect of CKD makes this patient group more comparable to the general population with diabetes where there is strong evidence for a positive effect of CGM on glycemic control. A metaanalysis of patients with both type 1 and type 2 diabetes (non-CKD) found 12 studies ( $n=1,276$; mostly observational) using the FreeStyle Libre to evaluate changes in $\mathrm{HbA}_{1 \mathrm{c}}$ after 2-4 months of use. The overall change in $\mathrm{HbA}_{1 \mathrm{c}}$ was $-0.56 \%$ (95\% CI: -0.76 to $-0.36, p<0.0001$ ) [17].

Another meta-analysis focused on patients with type 2 diabetes compared CGM with SMBG. The analyses included randomized studies with a duration of at least 12 weeks. The use of CGM (not including the FreeStyle Libre) was associated with an improvement of $\mathrm{HbA}_{1 \mathrm{c}}$ for CGM compared with SMBG of $-0.28 \%$ (95\% CI: -0.43 to $-013, p<0.01)$ [18]. For patients reaching CKD stages 4-5 (not on dialysis), the impact of renal failure makes these subgroups of the CKD spectrum more difficult to assess in terms of the effect CGM can have on glycemic control. No studies have specifically assessed the effect of CGM in CKD stage 4-5, but this group will mostly likely benefit from the use of CGM in line with previously reported studies.

\section{Conclusion}

The value of using $\mathrm{HbA}_{1 \mathrm{c}}$ as a glycemic marker remains unclear for patients on dialysis. Glycated albumin is a promising glycemic marker that could complement the use of $\mathrm{HbA}_{1 \mathrm{c}}$. No universal reference interval is accepted, which limits clinical use. CGM measures interstitial glucose levels avoiding the pitfalls of $\mathrm{HbA}_{1 \mathrm{c}}$ and glycated albumin. In addition, valuable information on time in hypo-, normo-, and hyperglycemia can be obtained. CGM could potentially improve glycemic control in subjects with CKD and patients receiving dialysis. CGM does have some limitations: These are primarily related to the cost and the practical challenges of using CGM in dialysis patients.

\section{Conflict of Interest Statement}

The authors have no conflicts of interest to declare.

\section{Funding Sources}

The authors have no funding sources to declare.

\section{Author Contributions}

Tobias Bomholt concepted the mini-review and analyzed relevant studies for inclusion, drafted and revised the article, provided intellectual content of critical importance to the work described, and approved the final version. Therese Adrian concepted the mini-review and analyzed relevant studies for inclusion, drafted and revised the article, provided intellectual content of critical importance to the work described, and approved the final version. Kirsten Nørgaard concepted the mini-review and analyzed relevant studies for inclusion, drafted and revised the article, provided intellectual content of critical importance to the work described, and approved the final version. Ajenthen G. Ranjan concepted the mini-review and analyzed relevant studies for inclusion, drafted and revised the article, provided intellectual content of critical importance to the work described, and approved the final version. Thomas Almdal analyzed relevant studies for inclusion, drafted and revised the article, provided intellectual content of critical importance to the work described, and approved the final version. Anders Larsson analyzed relevant studies for inclusion, drafted and revised the article, provided intellectual content of critical importance to the work described, and approved the final version. Mette Vadstrup concepted the mini-review and analyzed relevant studies for inclusion, drafted and revised the article, provided intellectual content of critical importance to the work described, and approved the final version. Marianne Rix concepted the mini-review and analyzed relevant studies for inclusion, drafted and revised the article, provided intellectual content of critical importance to the work described, and approved the final version. Bo Feldt-Rasmussen concepted the mini-review and analyzed relevant studies for inclusion, drafted and revised the article, provided intellectual content of critical importance to the work described, and approved the final version. Mads Hornum concepted the mini-review and analyzed relevant studies for inclusion, drafted and revised the article, provided intellectual content of critical importance to the work described, and approved the final version.

\section{References}

1 Nathan DM, Kuenen J, Borg R, Zheng H, Schoenfeld D, Heine RJ. Translating the A1C assay into estimated average glucose values. Diabetes Care. 2008;31(8):1473-8.

2 Peacock TP, Shihabi ZK, Bleyer AJ, Dolbare EL, Byers JR, Knovich MA, et al. Comparison of glycated albumin and hemoglobin $\mathrm{A}(1 \mathrm{c})$ levels in diabetic subjects on hemodialysis. Kidney Int. 2008;73(9):1062-8.
Bomholt et al. 
3 Riveline JP, Teynie J, Belmouaz S, Franc S, Dardari D, Bauwens M, et al. Glycaemic control in type 2 diabetic patients on chronic haemodialysis: use of a continuous glucose monitoring system. Nephrol Dial Transplant. 2009;24(9):2866-71.

4 Vos FE, Schollum JB, Coulter CV, Doyle TC, Duffull SB, Walker RJ. Red blood cell survival in long-term dialysis patients. Am J Kidney Dis. 2011 [cited 2018 Mar 16];58(4):591-8.

5 Oei E, Samad N, Visser A, Chowdhury TA, Fan SL. Use of continuous glucose monitoring in patients with diabetes on peritoneal dialysis: poor correlation with HbA1cand high incidence of hypoglycaemia. Diabet Med. 2016;33(9):e17-20.

6 KDIGO. KDIGO clinical practice guideline on diabetes mangement in chronic kidney disease [Internet]. 2019. Available from: https: //kdigo.org/wp-content/uploads/2020/ 10/KDIGO-2020-Diabetes-in-CKD-GL.pdf

7 Vernon Roohk H, Zaidi AR. A review of glycated albumin as an intermediate glycation index for controlling diabetes. J Diabetes Sci Technol. 2008;2(6):1114-21.

8 Yajima T, Yajima K, Hayashi M, Takahashi H, Yasuda K. Serum albumin-adjusted glycated albumin as a better indicator of glycemic control in Type 2 diabetes mellitus patients with short duration of hemodialysis. Diabetes Res Clin Pract. 2017;130:148-53.

9 Koga M, Kasayama S, Kanehara H, Bando Y. CLD (chronic liver diseases)-HbA1C as a suitable indicator for estimation of mean plasma glucose in patients with chronic liver diseases. Diabetes Res Clin Pract. 2008;81(2): 258-62.

10 Okada T, Nakao T, Matsumoto H, Nagaoka $\mathrm{Y}$, Tomaru R, Iwasawa $\mathrm{H}$, et al. Influence of proteinuria on glycated albumin values in diabetic patients with chronic kidney disease. Intern Med. 2011;50(1):23-9.
11 Jeppsson JO, Kobold U, Barr J, Finke A, Hoelzel W, Hoshino T, et al. Approved IFCC reference method for the measurement of $\mathrm{HbAlc}$ in human blood. Clin Chem Lab Med. 2002 Jan 29 [cited 2020 Jul 23];40(1):78-89.

12 Wu WC, Ma WY, Wei JN, Yu TY, Lin MS, Shih SR, et al. Serum glycated albumin to guide the diagnosis of diabetes mellitus. PLoS One. 2016;11(1):e0146780-14.

13 Freitas PAC, Ehlert LR, Camargo JL. Glycated albumin: a potential biomarker in diabetes. Arch Endocrinol Metab. 2017;61(3):296-304.

14 Battelino T, Danne T, Bergenstal RM, Amiel SA, Beck R, Biester T, et al. Clinical targets for continuous glucose monitoring data interpretation: recommendations from the international consensus on time in range. Diabetes Care. 2019 Aug;42(8):1593-603.

15 Képénékian L, Smagala A, Meyer L, Imhoff O, Alenabi F, Serb L, et al. Continuous glucose monitoring in hemodialyzed patients with type 2 diabetes: a multicenter pilot study. Clin Nephrol. 2014 Oct [cited 2019 Oct 25];82(4): 240-6.

16 Joubert M, Fourmy C, Henri P, Ficheux M, Lobbedez T, Reznik Y. Effectiveness of continuous glucose monitoring in dialysis patients with diabetes: the DIALYDIAB pilot study. Diabetes Res Clin Pract. 2015 Mar [cited 2019 Oct 25];107(3):348-54.

17 Evans M, Welsh Z, Ells S, Seibold A. The impact of flash glucose monitoring on glycaemic control as measured by HbAlc: a meta-analysis of clinical trials and real-world observational studies. Diabetes Ther. 2020;11(1):8395.
18 Dicembrini I, Mannucci E, Monami M, Pala L. Impact of technology on glycaemic control in type 2 diabetes: a meta-analysis of randomized trials on continuous glucose monitoring and continuous subcutaneous insulin infusion. Diabetes Obes Metab. 2019;21(12): 2619-25.

19 Abbott. Welcome to your freestyle libre system in-service guide. 2017. p. 22. Available from: https://www.myfreestyle.com/provid$\mathrm{er} / \mathrm{sites} / \mathrm{all} /$ themes/provider20/pdf/FreeStyle-Libre-In-Service-Guide.pdf

20 Dexcom. User guide. Available from: https:// s3-us-west-2.amazonaws.com/dexcompdf/ G6-CGM-Users-Guide.pdf

21 Medtronic. User guide Ipro2 [Internet]. 2016 [cited 2020 May 28]. Available from: https:// www.medtronicdiabetes.com/sites/default/ files/library/download-library/user-guides/ iPro2-with-Enlite-User-Guide.pdf

22 Medtronic. Envision $^{\mathrm{TM}}$ recorder: user guide [Internet]. Available from: https://fccid.io/ $\mathrm{OH} 27781 /$ User-Manual/Users-Manual-1--4342191.pdf

23 Medtronic. Envision ${ }^{\mathrm{TM}}$ PRO CGM|medtronic HCP portal [Internet]. 2020 [cited 2020 Jun 1]. Available from: https://hcp.medtronic-diabetes.co.uk/envision-pro-cgm

24 Medtronic. Guardian connect: system user guide [Internet]. Medtronic. 2017 [cited 2020 Jan 30]. Available from: https://www. medtronicdiabetes.com/sites/default/files/library/download-library/user-guides/guardian-connect-v3_2/us-manual.pdf

25 Eversense XL. Brugervejledning [Internet]. [cited 2019 Nov 22]. Available from: https:// global.eversensediabetes.com/sites/default/ files/2019--09/LBL-1402--08--101_Rev_A Eversense_User_Guide_mmol_DA_pressquality.pdf 